\title{
Study of an intrinsically safe infrastructure for training and research on nuclear technologies
}

\author{
Marco Ripani ${ }^{1, a}$, Stefano Frambati ${ }^{2}$, Luigi Mansani ${ }^{2}$, Maurizio Bruzzone ${ }^{2}$, Marco Reale ${ }^{2}$, \\ Stefano Monti ${ }^{3, \mathrm{~b}}$, Marco Ciotti ${ }^{4}$, Massimo Barbagallo ${ }^{5}$, Nicola Colonna ${ }^{5}$, Andrea Celentano ${ }^{1}$, \\ Mikhail Osipenko ${ }^{1}$, Giovanni Ricco ${ }^{1}$, Paolo Saracco ${ }^{1}$, Carlo Maria Viberti ${ }^{1}$, Oscar Frasciello ${ }^{6}$, \\ Pasquale Boccaccio $^{7}$, Juan Esposito ${ }^{7}$, Augusto Lombardi ${ }^{7}$, Mario Maggiore ${ }^{7}$, Leandro A. C. Piazza ${ }^{7}$, \\ Gianfranco Prete ${ }^{7}$, Rosa $\mathrm{Alba}^{8}$, Luciano Calabretta ${ }^{8}$, Gianluigi Cosentino ${ }^{8}$, Antonio Del Zoppo ${ }^{8}$, \\ Alessia Di Pietro ${ }^{8}$, Pierpaolo Figuera ${ }^{8}$, Paolo Finocchiaro ${ }^{8}$, Cettina Maiolino $^{8}$, Domenico Santonocito ${ }^{8}$, \\ Maria Schillaci ${ }^{8}$, Davide Chiesa ${ }^{9}$, Massimiliano Clemenza ${ }^{9}$, Ezio Previtali ${ }^{9}$, Monica Sisti ${ }^{9}$, \\ Alexander Kostyukov ${ }^{10}$, Antonio Cammi ${ }^{11}$, Sara Bortot ${ }^{11}$, Stefano Lorenzi ${ }^{11}$, Marco Ricotti ${ }^{11}$, \\ Sandra Dulla ${ }^{12}$, Piero Ravetto ${ }^{12}$, Guglielmo Lomonaco ${ }^{1,13}$, Alessandro Rebora ${ }^{13}$, Daniele Alloni ${ }^{14}$, \\ Andrea Borio di Tigliole ${ }^{14, c}$, Marcella Cagnazzo ${ }^{14}$, Riccardo Cremonesi ${ }^{14}$, Giovanni Magrotti ${ }^{14}$, \\ Sergio Manera ${ }^{14}$, Fabio Panza ${ }^{14}$, Michele Prata ${ }^{14}$ and Andrea Salvini ${ }^{14}$ \\ ${ }^{1}$ INFN, Sezione di Genova, via Dodecaneso 33, Genoa 16146, Italy \\ 2 Ansaldo Nucleare SpA, C.so F.M. Perrone 25, Genoa 16152, Italy \\ ${ }^{3}$ ENEA, via Martiri di Monte Sole, 4, Bologna 40129, Italy \\ ${ }^{4}$ ENEA, via Enrico Fermi, 45, Frascati (Rome) 00044, Italy \\ ${ }^{5}$ INFN, Sezione di Bari, via E. Orabona n. 4, Bari 70125, Italy \\ ${ }^{6}$ INFN, Laboratori Nazionali di Frascati, via Enrico Fermi, 40, Frascati (Rome) 00044, Italy \\ ${ }^{7}$ INFN Laboratori Nazionali di Legnaro, Viale dell'Università 2, Legnaro (Padova) 35020, Italy \\ ${ }^{8}$ INFN Laboratori Nazionali del Sud, via S. Sofia 62, Catania 95125, Italy \\ ${ }^{9}$ INFN, Sezione di Milano Bicocca e Università di Milano Bicocca, Piazza dell'Ateneo Nuovo, 1, \\ Milan 20126, Italy \\ ${ }^{10}$ Lomonosov Moscow State University, GSP-1, Leninskie Gory, Moscow 119991, Russian Federation \\ ${ }^{11}$ Politecnico di Milano, Piazza L. da Vinci, 32, Milan 20133, Italy \\ ${ }^{12}$ Politecnico di Torino, Corso Duca degli Abruzzi, 24, Turin 10129, Italy \\ ${ }^{13}$ DIME - Università di Genova, via all'Opera Pia, 15/A, Genoa 16145, Italy \\ ${ }^{14}$ Università di Pavia and LENA, via Aselli 41, Pavia 27100, Italy
}

\begin{abstract}
Within European Partitioning \& Transmutation research programs, infrastructures specifically dedicated to the study of fundamental reactor physics and engineering parameters of future fast-neutron-based reactors are very important, being some of these features not available in present zero-power prototypes. This presentation will
\end{abstract}

\footnotetext{
${ }^{\text {a }}$ Corresponding author: ripani@ge.infn.it

${ }^{\mathrm{b}}$ Currently at IAEA, Wagramer Straße 5, Vienna 1220, Austria

${ }^{\mathrm{c}}$ Currently at IAEA, Wagramer Straße 5, Vienna 1220, Austria
}

This is an Open Access article distributed under the terms of the Creative Commons Attribution License 4.0, which permits unrestricted use, distribution, and reproduction in any medium, provided the original work is properly cited. 
illustrate the conceptual design of an Accelerator-Driven System with high safety standards, but ample flexibility for measurements. The design assumes as base option a $70 \mathrm{MeV}$, $0.75 \mathrm{~mA}$ proton cyclotron, as the one which will be installed at the INFN National Laboratory in Legnaro, Italy and a Beryllium target, with Helium gas as core coolant. Safety is guaranteed by limiting the thermal power to $200 \mathrm{~kW}$, with a neutron multiplication coefficient around 0.94 , loading the core with fuel containing Uranium enriched at $20 \%$ inserted in a solid-lead diffuser. The small decay heat can be passively removed by thermal radiation from the vessel. Such a system could be used to study, among others, some specific aspects of neutron diffusion in lead, beam-core coupling, target cooling and could serve as a training facility.

\section{Introduction}

Operation of the widespread thermal nuclear reactors results in the accumulation of important quantities of highly radioactive, highly toxic, long-lived nuclear materials, in the form of plutonium, Minor Actinides (MA) and Long-Lived Fission Products (LLFP). In the European Union, annually, about 2500 tons of spent fuel are produced, of which about 25 tons are plutonium isotopes, 3.5 tons are minor actinides and about 3 tons are long lived fission products. In some countries, spent fuel is reprocessed and used for the fabrication of mixed oxide (MOX) fuels, however with limited impact on the continuous build-up of these materials in storage sites.

Different solutions have been proposed and minimization of waste has become an important aspect in the development of innovative nuclear energy systems (see, as examples, [1-4]). One of the possible strategies for waste minimization implies the so-called double-strata approach [5], in which the second stratum is a transuranic $(\mathrm{Pu}, \mathrm{MA})$ transmutation scheme based on dedicated fast reactors and Accelerator-Driven Systems (ADS) ${ }^{1}$.

In fact, many MA produced in reactor operation like ${ }^{237} \mathrm{~Np},{ }^{241} \mathrm{Am},{ }^{244} \mathrm{Cm}$, have a fission cross section threshold of about $0.5 \mathrm{MeV}$. Therefore a "fast" spectrum can lead to burning MA via fission quite effectively due to the high density of energetic neutrons. Here "fast" refers to the fact that, on the contrary to what happens in a "thermal" reactor, neutrons are not slowed down by a moderator inside the reactor core, thus maintaining a higher average energy (i.e. a higher velocity). The more energetic neutron spectrum is obtained by using as a coolant a medium or heavy element, such as sodium or lead, or a low density gas. As a result, in fast reactors much of the neutron spectrum is above several hundred keV. For this and other reasons specifically related to the choice of cooling medium, among innovative nuclear reactor concepts, lead-cooled fast systems are of particular interest and many international research projects are focused on this technology, e.g. ELSY [6-8], CDT [9] and LEADER [10].

Safety aspects of fast critical reactors do not allow to load the core with MA beyond certain limits, due to the relatively small fraction of delayed neutrons (essential for reactor control) in the actinide fission process [11]. Accelerator-Driven Systems (ADS) could instead be particularly well-suited to maximize the transmutation rate, while still operating in a highly safe regime [12]. The main difference between a nuclear reactor and an ADS is that in the first case the effective multiplication coefficient is kept equal to unity, thereby ensuring that the fission chain reaction is self-sustained, while ADS are characterized by an effective multiplication coefficient smaller than unity (typically 0.95-0.98). Thus, to maintain a steady state condition of operation in an ADS, additional neutrons must be supplied by an external neutron source, by using an accelerated particle beam impinging on a neutron production target. Most ADS designs are based on a fast reactor core, modified to obtain multiplication coefficients less than unity. For a high-power ADS, accelerator requirements are rather stringent: high neutron production

\footnotetext{
${ }^{1}$ Italy is deeply involved on one side in planning a IV generation Lead-cooled fast reactor demonstrator, Alfred, on the other side to develop the knowledge basis for achieving the needed degree of confidence with ADS systems.
} 
rate; high beam power (high energy and/or current); very high stability i.e. very few beam trips during long running times; minimal electric power consumption i.e. minimal ratio between actual electric power consumption and the outlet power transferred to the beam (from 4 to 25 in existing accelerators). Most of these requirements are more severe than in conventional research accelerators and require, at least for a high-power ADS, a special design.

Another important aspect in the framework of innovative nuclear energy system projects is the opportunity to use infrastructures for research and study where experimental tests on new concepts can be performed, in order to validate measurement methodologies, simulation codes and data libraries, as well as to improve our understanding of the complex dynamic and kinetic effects in fast-neutron heavymetal-cooled sub-critical systems. Therefore also in ADS design, lead technology is present in several projects: PDS-XADS [13], IP-EUROTRANS [14-16], Guinevere [17], MYHHRA [18] and EFIT [19]. The Guinevere setup at SCK · CEN in Mol, Belgium [17], based on a D+T accelerator providing nearly monochromatic $14 \mathrm{MeV}$ neutrons embedded in a solid Lead matrix started operations in 2011.

Among the basic R\&D requirements are the capability to test and develop experimental methods for the on-line measurement of sub-criticality in ADS systems and the need for hands-on experience on the kinetic and dynamic behaviour in fast systems. Such an experience is essential in order to validate our theoretical understanding of the main processes and parameters underlying fast neutron systems, but it is also fundamental to assess the potential impact of these effects on control and safety parameters. It is of paramount importance to develop and build facilities powerful enough to fulfill a majority of these requests, but sufficiently low-power to not overcome the zone of comfortable, high-safety operation.

Following these requirements, a proposal was put forward by INFN, in collaboration with Ansaldo Nucleare, ENEA, Politecnico di Milano, Politecnico di Torino, University of Genova and University of Pavia - LENA [20]. Besides the motivations outlined above, the proposal was inspired by the availability in the very near future of the proton cyclotron purchased by INFN as driver for the SPES project on radioactive ion beams [21]. The main features of the proposal are described in Sect. 4.

\section{Nuclear waste transmutation}

Transmutation (or nuclear incineration) of radioactive waste can take place due to neutron-induced reactions that transform long-lived radioactive isotopes into stable or short-lived isotopes. In the case of Long Lived FP (LLFP) like e.g. ${ }^{151} \mathrm{Sm},{ }^{99} \mathrm{Tc},{ }^{121} \mathrm{I},{ }^{79} \mathrm{Se}$, etc. transmutation can occur via neutron radiative capture $(\mathrm{n}, \gamma)$ like e.g. in the reaction $\mathrm{n}+{ }^{99} \mathrm{Tc}(2.1 \times 105 \mathrm{y}) \rightarrow{ }^{100} \mathrm{Tc}(16 \mathrm{~s}) \rightarrow{ }^{100} \mathrm{Ru}$ (stable). For Plutonium and MA like e.g. ${ }^{240} \mathrm{Pu},{ }^{237} \mathrm{~Np},{ }^{241,243} \mathrm{Am},{ }^{244,245} \mathrm{Cm}$, etc., transmutation can occur via either neutron-induced fission (n,f) or neutron capture $(\mathrm{n}, \gamma)$. Apart for ${ }^{245} \mathrm{Cm}$, MA are characterized by a fission threshold around the $\mathrm{MeV}$ neutron energy. Such isotopes could efficiently be burned in fast reactors, where the neutron spectrum typically ranges from $10 \mathrm{keV}$ to $10 \mathrm{MeV}$, but due to core reaction dynamic control requirements, only small amounts of these elements can be inserted in the reactor. An important remark to be made is that, due to the sub-criticality of the system, in an ADS delayed neutrons are less relevant for reactor control [22], since the kinetics is dominated by prompt neutrons which follow the time-behaviour of the external neutron source (at least for a quite energetic source). Therefore a fast ADS offers a more ample capability in terms of adding Transuranic elements to the fuel and burning them.

\section{A low-power ADS}

The typical layout of an ADS is shown in Fig. 1. Electrons, protons or other ions are accelerated by a specific particle accelerator. The accelerated beam is then transported to a subcritical reactor core, i.e. a core for which the effective neutron multiplication coefficient $\mathrm{k}_{\text {eff }}$ is less than unity. The fact that $\mathrm{k}_{\mathrm{eff}}<1$ means that at each fission, the number of neutrons that will in turn produce another fission is less 


\section{EPJ Web of Conferences}
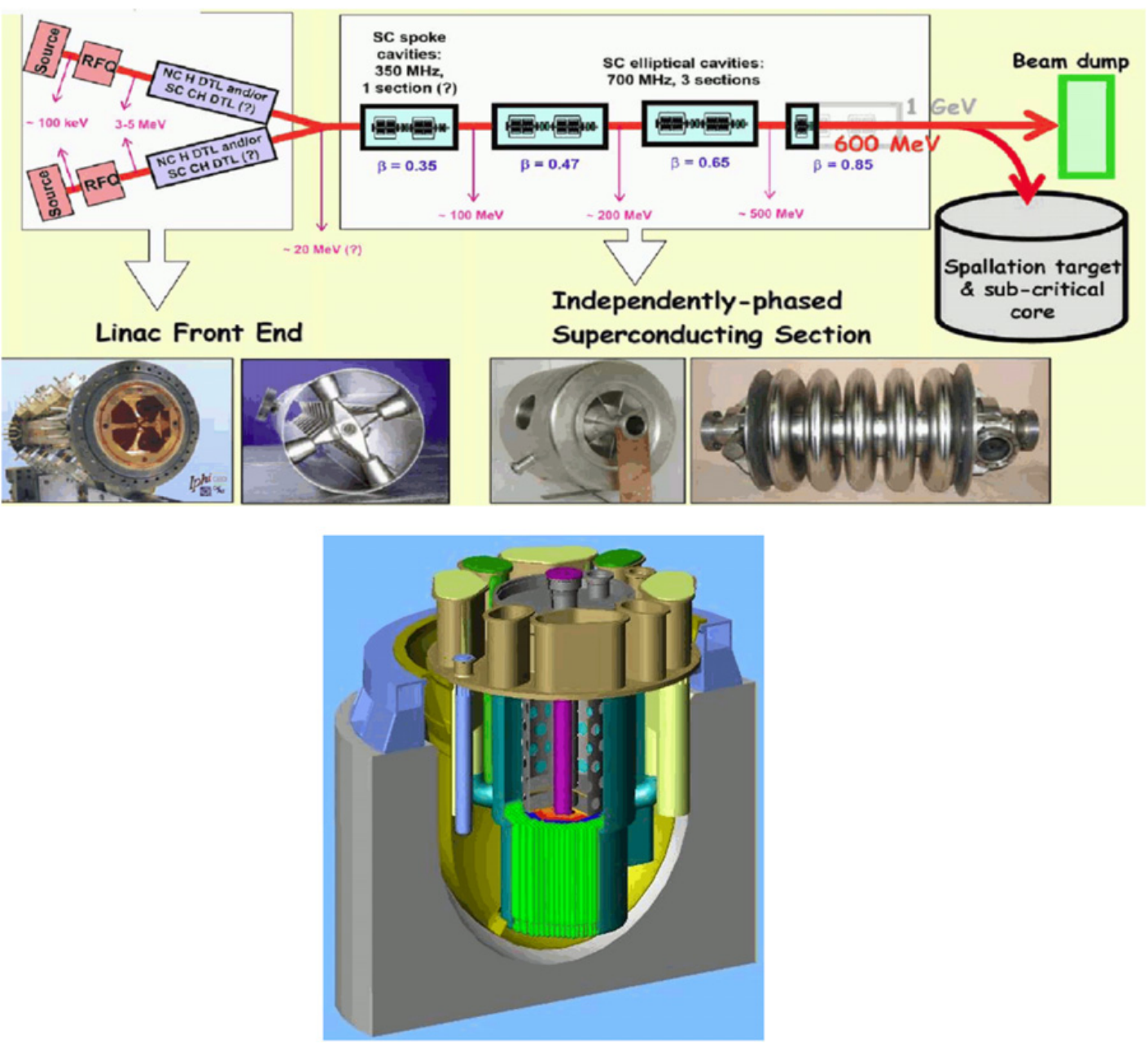

Figure 1. Typical ADS layout, comprising an accelerator (in this case a proton accelerator), a beam transport system and a subcritical reactor core to which the beam is coupled. Pictures are taken from the EFIT project 19.

than unity, which implies that there is no self-sustained chain reaction. As a consequence, the reactor can be operated in steady state conditions only if additional neutrons are supplied through an external source. External neutron production is obtained by driving the beam into an appropriate target, where the beam is completely absorbed and undergoes a number of nuclear interactions with free neutrons appearing as reaction products.

In this proposal, the $70 \mathrm{MeV}, 0.75 \mathrm{~mA}$ proton cyclotron purchased by INFN as driver for the SPES project on radioactive ion beams at INFN National Laboratory of Legnaro (LNL) [21] was taken as reference accelerator. For the core design, $\mathrm{UO}_{2}$ with $20 \mathrm{wt} \%{ }^{235} \mathrm{U}$ was chosen as the fuel, to avoid security issues related to handling $\mathrm{Pu}$. The fuel rods and assemblies were arranged in such a way to guarantee a neutron multiplication coefficient $\sim 0.95$, which is the limit for waste storage facilities. The resulting thermal power was required to be in the order of $150-200 \mathrm{~kW}$, sufficiently low to limit safety issues but sufficiently high to study some aspects of dynamics. The corresponding core temperature should not exceed $300^{\circ} \mathrm{C}$, which allows to use a solid Lead matrix being well below the melting point (however, to ensure mechanical stability, the Lead matrix would be embedded in a steel structure). This 
$3^{\text {rd }}$ European Energy Conference
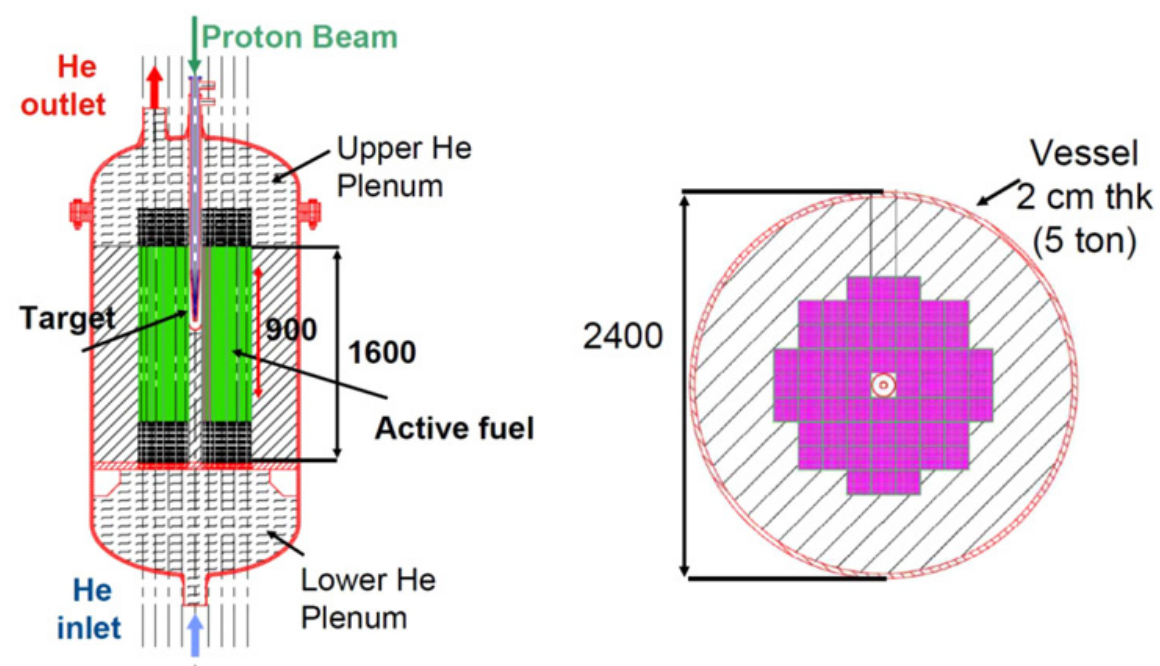

Figure 2. Overview of the proposed facility. The lead radial reflector is not to scale in order to improve image readability. Left: vertical section of the facility, with the core $(160 \mathrm{~cm}$ of height, $90 \mathrm{~cm}$ of which containing active fuel), lower and upper helium plena and inlet/outlet channels, axial and radial reflectors. The proton beam enters from the vessel upper head. Right: horizontal section of the active core, with 60 active elements, the radial reflector and the central convertor position.

simplifies engineering aspects further as no liquid metal circulation system has to be designed. Cooling of the core would be performed by circulating He gas through small channels placed between the fuel elements. As convertor to produce the neutrons from the incident $70 \mathrm{MeV}$ proton beam, Beryllium was chosen: the presence of a weakly bound neutron provides abundant neutron production at this relatively low beam energy, in the order of 0.1 neutrons per incident proton.

A dedicated measurement of the neutron yield from Beryllium at the similar energy of $62 \mathrm{MeV}$ was performed by an INFN team at Laboratori Nazionali del Sud in Catania, Italy [22, 24]. Figure 2 shows a vertical and horizontal section of the vessel containing the active core, with the convertor target situated approximately at the centre of the core and the inlet for the incident proton beam. The geometry of the beryllium target has been derived from the conical shape already used for the TRADE project [25]. The conical surface allows the distribution of the beam power on a wide surface, thus helping target cooling. Beryllium was chosen both because it provides a very good proton-to-neutron conversion factor (mainly due to $(\mathrm{n}, \mathrm{xn})$ reactions on the ${ }^{9} \mathrm{Be}$ isotope), and due to its high thermal conductivity, about 6 times that of lead. The about $50 \mathrm{~kW}$ total thermal power deposited on the target by the beam would be removed by a dedicated He gas cooling channel.

Figure 3 shows the neutron spectrum in an inner fuel rod simulated with the MCNPX code [26], together with some representative fission cross sections for minor actinides. The initial value of $\mathrm{k}_{\mathrm{eff}}$ is $0.942 \pm 0.001$ (100 pcm uncertainty). The total evaluated reactor power with its systematic error is $\mathrm{P}_{\text {tot }}=199 \mathrm{~kW}_{\text {th }}$, i.e. about $0.4 \mathrm{~W}_{\mathrm{th}} / \mathrm{cm}^{3}$, with a maximum uncertainty of about $15 \%$, depending on the libraries used.

Once the system was characterized and the best positions for MA and FP transmutations evaluated, the burn-up of some "test rods" was investigated at nominal conditions. In order to perform a quite comprehensive evaluation, a single burn-up calculation was performed, positioning 4 "test" rods inside the core, considering both a MA mixture and other types of fuel (Fig. 4). Here only results for Fuel 3 and 5 will be reported, both comprising a Full Actinide CerCer rod (see Table 1), placed at a position 


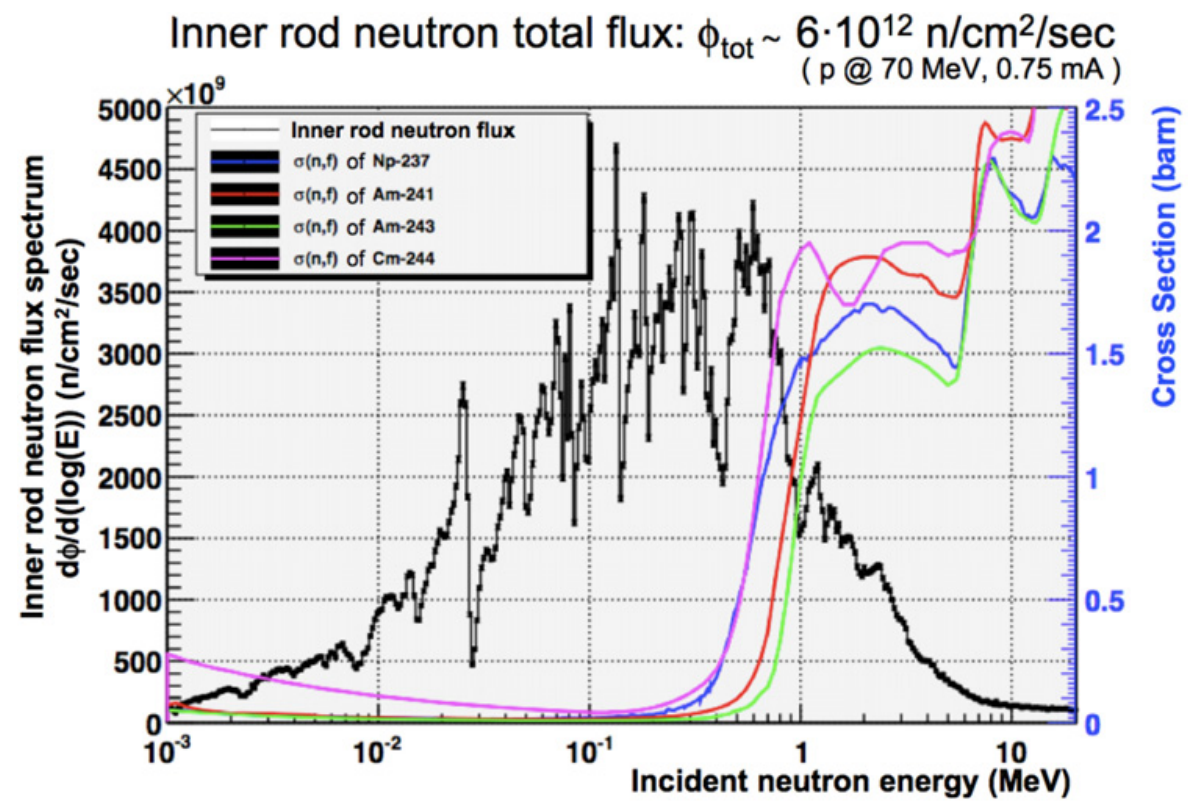

Figure 3. Inner rod neutron flux spectrum compared with the fission neutron cross-section in MA. The integrated flux is around $6 \cdot 10^{12} \mathrm{n} / \mathrm{cm}^{2} / \mathrm{sec}$ while the flux useful for fast fission (i.e. above $0.5 \mathrm{MeV}$ ) amounts to $30 \%$ of the integrated flux.

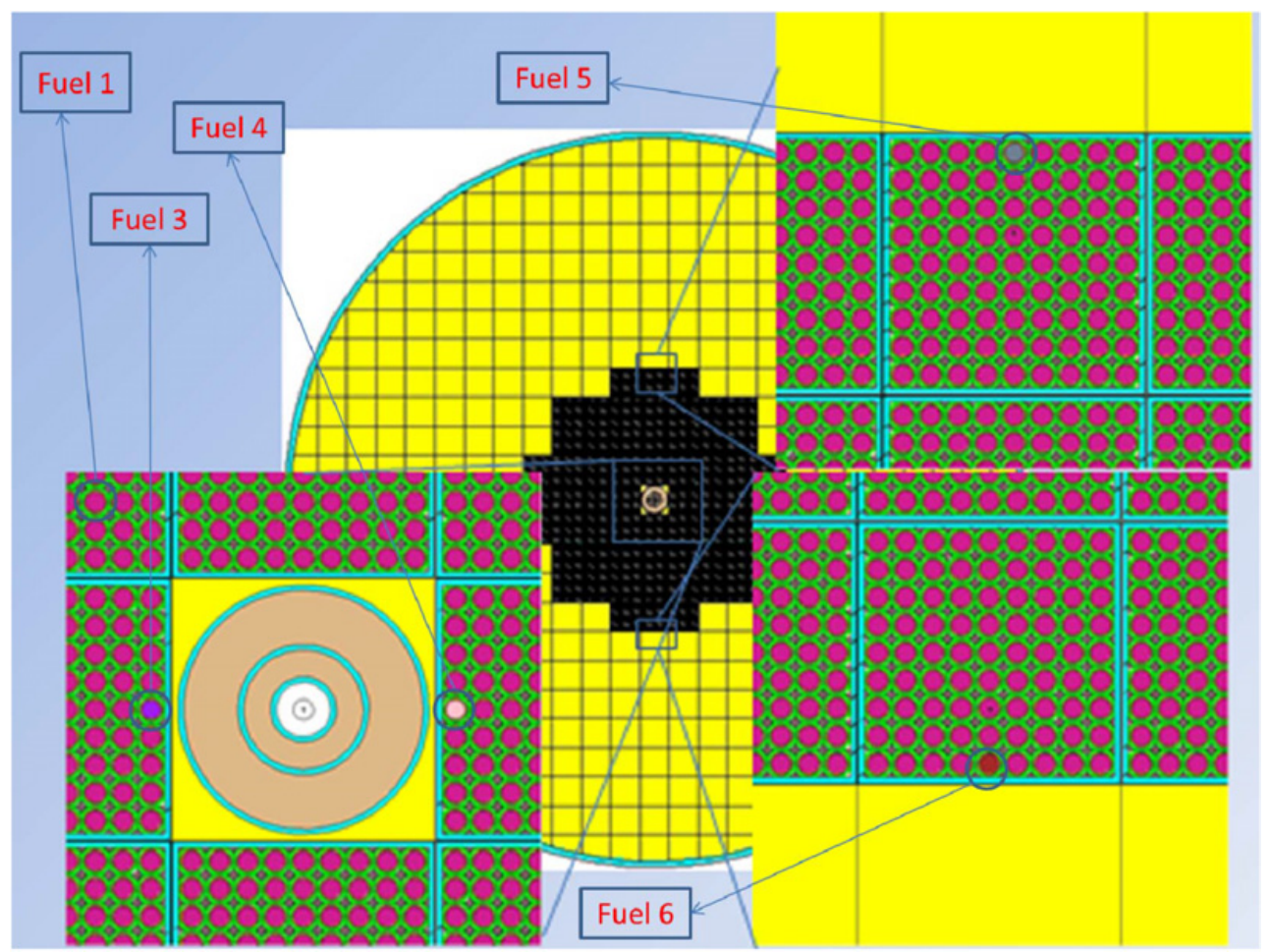

Figure 4. Core radial section with 3 zooms highlighting (in different colours) the 4 "test" rod positions. 
Table 1. Masses [g] for various actinides (at different times) for fuels 3 and 5.

\begin{tabular}{|l|c|c|c|c|c|}
\hline & Fresh Fuels 3 \& 5 & \multicolumn{2}{|c|}{ Fuel 3 } & \multicolumn{2}{c|}{ Fuel 5 } \\
\hline & & $\begin{array}{c}\text { (1y after } \\
\text { irradiation) }\end{array}$ & $\begin{array}{c}(10 \mathrm{y} \text { after } \\
\text { irradiation) }\end{array}$ & $\begin{array}{c}\text { (1y after } \\
\text { irradiation) }\end{array}$ & $\begin{array}{c}\text { (10y after } \\
\text { irradiation) }\end{array}$ \\
\hline $\mathrm{Np}^{237}$ & - & $6.79471 \mathrm{e}+1$ & $7.01532 \mathrm{e}+1$ & $6.79471 \mathrm{e}+1$ & $7.01532 \mathrm{e}+1$ \\
\hline $\mathrm{Np}^{239}$ & - & $8.86555 \mathrm{e}-6$ & $8.85805 \mathrm{e}-6$ & $8.86555 \mathrm{e}-6$ & $8.85805 \mathrm{e}-6$ \\
\hline $\mathrm{Pu}^{238}$ & - & $4.73037 \mathrm{e}-2$ & $4.68773 \mathrm{e}-2$ & $3.57616 \mathrm{e}-2$ & $3.53688 \mathrm{e}-2$ \\
\hline $\mathrm{Pu}^{239}$ & - & $2.16127 \mathrm{e}-3$ & $5.53281 \mathrm{e}-3$ & $2.15127 \mathrm{e}-3$ & $5.52281 \mathrm{e}-3$ \\
\hline $\mathrm{Pu}^{240}$ & - & $5.83828 \mathrm{e}-2$ & $2.47827 \mathrm{e}-1$ & $5.83828 \mathrm{e}-2$ & $2.47827 \mathrm{e}-1$ \\
\hline $\mathrm{Pu}^{241}$ & - & $2.29209 \mathrm{e}-5$ & $8.86302 \mathrm{e}-5$ & $2.34927 \mathrm{e}-5$ & $8.90010 \mathrm{e}-5$ \\
\hline $\mathrm{Pu}^{242}$ & - & $6.90999 \mathrm{e}-3$ & $6.90988 \mathrm{e}-3$ & $5.13000 \mathrm{e}-3$ & $5.12991 \mathrm{e}-3$ \\
\hline $\mathrm{Am}^{241}$ & $1.57 \mathrm{e}+2$ & $1.56749 \mathrm{e}+2$ & $1.54505 \mathrm{e}+2$ & $1.56749 \mathrm{e}+2$ & $1.54505 \mathrm{e}+2$ \\
\hline $\mathrm{Am}^{243}$ & $1.03 \mathrm{e}+1$ & $1.02990 \mathrm{e}+1$ & $1.02903 \mathrm{e}+1$ & $1.02990 \mathrm{e}+1$ & $1.02903 \mathrm{e}+1$ \\
\hline $\mathrm{Cm}^{242}$ & - & $3.06488 \mathrm{e}-3$ & $2.58117 \mathrm{e}-9$ & $2.24053 \mathrm{e}-3$ & $1.88693 \mathrm{e}-9$ \\
\hline $\mathrm{Cm}^{243}$ & $1.88 \mathrm{e}-2$ & $1.77716 \mathrm{e}-2$ & $1.43425 \mathrm{e}-2$ & $1.77716 \mathrm{e}-2$ & $1.43425 \mathrm{e}-2$ \\
\hline $\mathrm{Cm}^{244}$ & $7.18 \mathrm{e}-1$ & $6.61189 \mathrm{e}-1$ & $4.68427 \mathrm{e}-1$ & $6.61189 \mathrm{e}-1$ & $4.68427 \mathrm{e}-1$ \\
\hline $\mathrm{Cm}^{245}$ & $1.26 \mathrm{e}-1$ & $1.25989 \mathrm{e}-1$ & $1.25897 \mathrm{e}-1$ & $1.25989 \mathrm{e}-1$ & $1.25897 \mathrm{e}-1$ \\
\hline
\end{tabular}

close to the target (3, "inner" rod) or at the periphery of the core (5, "outer" rod). Further data and results can be found in [27].

The apparatus studied here is not a full-scale ADS, whose purpose would be to maximize waste incineration such as to reach the ore-level radiotoxicity in a shorter time scale of the order of few hundred years. It is instead devoted to investigate the feasibility and effectiveness of transmutation processes in a laboratory time scale of the order of a few years. Given this distinction, we performed the burn-up calculations considering one year of waste irradiation inside the ADS and 10 years of measurements.

Several values of $\mathrm{k}_{\text {eff }}$ were calculated up to 2145 EFPD (Equivalent Full Power Days) in the geometrical conditions of Fig. 4. However, in order to consider a more "realistic" scenario, the burn-up calculations have been limited to an EOL (End Of Life) of 410 EFPD, using MONTEBURNS [28], coupling MCNP5 and ORIGEN2 codes, with the ENDF/B-VII.1 libraries.

To give some further details on the obtained results, Table 1 reports, for fuels 3 and 5, the masses (in grams) for various actinides, evaluated before being loaded in the core, 1 and 10 years after the discharge. Looking at the table, $\mathrm{Cm}^{244}$ is substantially the only isotope that undergoes a significant reduction during irradiation (around 8\%): considering the well-known toughness in reducing that isotope inside critical reactors [1-3], the obtained results appear worthy of further future investigations.

The features of the accelerator and core considered here are obviously not suitable for massive transmutation purposes. However, these preliminary studies indicate that the proposed setup may be a demonstrator facility where to test the concepts and effectiveness of burn out of minor actinides in nuclear waste. Further studies are being conducted to assess in detail the capabilities of this apparatus and define its potentiality for an experimental program on lead fast systems and waste burn-out. An alternative version is also currently being studied, where the core would only contain MA (uraniumfree fuel) and Plutonium (to sustain the $\mathrm{k}_{\mathrm{eff}}$ ). The realistic measurement of transmutation efficiency for industrial applications will however require, as a natural evolution, an improved system with higher power (i.e. in the MW range) and liquid lead cooling. The proposed facility may be therefore seen as an intermediate step between the existing Guinevère apparatus [17] and the future MYRRHA [18 and EFIT [19]. 


\section{References}

[1] E. Bomboni, N. Cerullo, G. Lomonaco, Science and Technology of Nuclear Installations, vol. 2009, Article ID 193594, 14 pages. doi:10.1155/2009/193594

[2] G. Mazzini, E. Bomboni, N. Cerullo, E. Fridman, G. Lomonaco, E. Shwageraus, Science and Technology of Nuclear Installations, vol. 2009, Article ID 749736, 13 pages. doi:10.1155/2009/749736

[3] B. Vezzoni, N. Cerullo, G. Forasassi, E. Fridman, G. Lomonaco, V. Romanello, E. Shwageraus E., Science and Technology of Nuclear Installations, vol. 2009, Article ID 940286, 16 pages. doi:10.1155/2009/940286

[4] N. Cerullo, G. Lomonaco, in I. Crossland, Nuclear Fuel Cycle Science and Engineering, pp. 333395, Woodhead Publishing

[5] OECD/NEA, Advanced Nuclear Fuel Cycles and Radioactive Waste Management, Paris (2006)

[6] L. Cinotti et al., Proceedings of the ICAPP 2007 International Conference on Advances in Nuclear Power Plants, Nice, France, May 13-18, 2007

[7] A. Alemberti et al., Proceedings of the FISA 2009 Seventh European Commission conference on Euratom research and training in reactor systems, Prague, Czech Republic, June 22-24, 2009

[8] L. Cinotti et al., Journal of Nuclear Materials, 415 (2011)

[9] D. De Bruyn et al., Proceedings of the ICAPP 2010 International Conference on Advances in Nuclear Power Plants, San Diego, CA, USA, June 13-17, 2010

[10] "Lead-cooled European Advanced DEmonstrator Reactor", funded under the Seventh Framework Programme for Research of the European Commission, contract number 249668

[11] H. Nifenecker et al., Nuclear Instruments and Methods in Physics Research A 463, p. 428 (1999)

[12] OECD-NEA Report N. 3109, http://www.oecd-nea.org/ndd/reports/2002/3109/ (2002)

[13] L. Cinotti et al., Proceedings of the International Workshop on P\&T and ADS Development, Mol, Belgium, October 6-8, 2003

[14] G. Rimpault et al., CEA report SPRC/LEDC/R 10-2 (2010)

[15] D. De Bruyn et al., Proceedings of the Workshop on Technology and Components for Acceleratordriven Systems, Karlsruhe, Germany, March 15-17, 2010

[16] G. Granget et al., Proceedings of the Workshop on Technology and Components for Acceleratordriven Systems, Karlsruhe, Germany, March 15-17, 2010

[17] A. Billebaud et al., Proceedings of Global 2009 - International Conference GLOBAL 2009 "The Nuclear Fuel Cycle: Sustainable Options \& Industrial Perspectives", Paris, France; http://hal.in2p3.fr/docs/00/41/44/31/PDF/GLOBAL2009-AB-9414-final .pdf

[18] H. Aït Abderrahim et al., Nuclear Physics News 20, p. 24 (2010)

[19] L. Mansani et al., Journal of Nuclear Technology, 180, p. 241 (2012)

[20] G. Ricco et al. - ISR (Intrinsically Safe Reactor). Submitted to European Physical Journal - Plus, http://www.ge.infn.it/ opisso/CDR/Index.htm

[21] G. Prete, Proceedings of EURORIB 2012 - European Radioactive Ion Beam Conference 2012, Abano Terme (Padova), Italy, May 21-25 2012; http://agenda.infn.it/ conferenceDisplay · py? conf Id=3946

[22] P. Bosio et al., Kerntechnik, 66, p. 260 (2001)

[23] R. Alba et al., Proceedings of the $11^{\text {th }}$ International Conference on Nucleus-Nucleus collisions, San Antonio, Texas, USA, May 27-June 1 2012, Journal of Physics: Conference Series 420, 012162 (2013)

[24] M. Osipenko et al., Nuclear Instruments and Methods in Physics Research A 723, 8 (2013)

[25] R. Rosa, Proceedings of the Third Annual Workshop on Accelerator-Driven Subcritical System (ADSS) Experiments, Idaho State University, Pocatello, Idaho, USA, June 1-2 2005; 
$3^{\text {rd }}$ European Energy Conference

http://www.iac.isu.edu/docs/workshops/beller/ADSS2005/Rosa\%20ID\%20ADSS\% 2005\%20TRADE.pdf

[26] D. B. Pelowitz, MCNPX User's Manual, Version 2.6.0, Los Alamos National Laboratory report LA-CP-07-1473 (2008)

[27] G. Lomonaco, O. Frasciello, M. Osipenko, G. Ricco, M. Ripani, ISR (Intrinsically Safe Reactor) - Section 10 Burnup and transmutation. Submitted to European Physical Journal - Plus

[28] D. I. Poston, H. R. Trellue, User's manual - Version 2.0 for MONTEBURNS Version 1.0, LAUR-99-4999 PSR-0455/01 (1999)

[29] E. Bomboni, N. Cerullo, G. Lomonaco, V. Romanello, Science and Technology of Nuclear Installations, vol. 2008, Article ID 265430, 18 pages. doi:10.1155/2008/265430 